Karlheinz Sonntag, Ekkehart Frieling, Ralf Stegmaier

\section{Lehrbuch Arbeitspsychologie}

Verlag Hans Huber, Bern et al., 3., vollst. überarbeitete Auflage 2012. ISBN 978-3-456-85002-3

Arbeitspsychologie und Arbeitswissenschaft sind untrennbar miteinander verknüpft. So ist es sicherlich nicht verwunderlich, dass gerade dieses, nunmehr zum dritten Mal vollständig überarbeitetes Lehrbuch der Arbeitspsychologie, besonders bei lehrenden und praktizierenden Arbeitswissenschaftlern auf großes Interesse stößt.

$\mathrm{Zu}$ erwähnen ist, dass bereits die Machart des Buches positiv auffällt. Durchgängig vierfarbig gedruckt, erleichtern die Farbgebung, die saubere Aufbereitung der Grafiken und Abbildungen sowie die Struktur des Buches einen Überblick über das umfangreich besprochene Thema zu behalten. Infoboxen geben detailliertere Informationen zu interessanten Hintergründen.

Das Buch ist in insgesamt fünf Teile untergliedert.

Der erste Teil umfasst die theoretischen Grundlagen der Arbeitspsychologie und erläutert das Selbstverständnis, die Geschichte und die Theoriebildung der Arbeitspsychologie.

Der zweite Teil vertieft die Methoden, Verfahren und Instrumente. Besonders werden hier Befragungen, Beobachtungen und Arbeitsanalysen besprochen, aber auch die wichtigen physiologischen Methoden sowie Simulationen und Experimente.

Der dritte Teil ist dem aktuellen Thema der personalen Voraussetzungen, deren Förderung und Erhalt gewidmet. Hierin wird auf Verhaltens- und
Leistungsdispositionen eingegangen, personale Förderung und Kompetenzentwicklung sowie Arbeit, Gesundheit und Wohlbefinden im Allgemeinen.

Der vierte Teil, der sich mit der Bewertung und Gestaltung von Arbeitstätigkeiten beschäftigt, umschreibt wichtige Basics der Arbeitswissenschaft, wie beispielsweise die humane Arbeitsgestaltung, die Gestaltung der Arbeitsumgebung und des Arbeitsplatzes sowie die Gestaltung organisationaler Bedingungen aus arbeitspsychologischer Sicht.

Der fünfte und damit letzte Teil dieses Lehrbuches beschäftigt sich mit Beispielen arbeitspsychologischen Handelns in Praxis und Forschung und ist ausgesprochen gut geeignet, als Lehrvorlage oder zum Selbststudium zu dienen. Vorgestellt werden die arbeitspsychologische Evaluation eines neuen Montagesystems, die Analyse und Förderung von Diagnosefähigkeiten in komplexen technischen Systemen sowie die Anforderungsanalyse und Kompetenzmodellierung nach Veränderungen.

Die Autoren betonen, dass sie kein spezifisches Lehrbuch für den Bachelorund/oder Masterstudiengang schreiben wollten. Sie möchten mit diesem Buch ein grundlegendes Lehrbuch für alle Studierenden der Psychologie, aber auch der Ingenieurwissenschaften, der Betriebswirtschaft, der Soziologie und der Bildungswissenschaften, zur Verfügung stellen. Zudem bieten sie das Buch interessierten Wissenschaftlern, Beratern und Praktikern an, die mit der Analyse und Gestaltung menschlicher und produktiver Arbeit betraut sind.

Für mich persönlich steht fest, dass dieses Buch meine berufliche aber auch meine Lehrtätigkeit begleiten und bereichern wird.

Yvonne Ferreira
Karl König

\section{Arbeit und Persönlichkeit. In- dividuelle und interprersonelle Aspekte}

Reihe Psychodynamik der Arbeitswelt. Bandes \& Apsel Verlag GmbH, Frankfurt, 2011. ISBN 978-3-86099-706-2

Die Reihe „Psychodynamik der Arbeitswelt" setzt sich zum Ziel, die Ursachen von Entfremdung in der Arbeitswelt zu ergründen und darüber aufzuklären. Sie wendet sich an einen weiten Personenkreis: Psychotherapeuten, Psychoanalytiker, Psychiater, Arbeitsmediziner und -psychologen, Supervisoren, Berater und Coaches, Gewerkschafter und Mitarbeiter der Agentur für Arbeit, Arbeits- und Sozialwissenschaftler, Juristen für Arbeitsrecht sowie alle Arbeitenden, die sich für die Arbeitswelt aus psychodynamischer Sicht interessieren.

Das vorliegende Buch beschäftigt sich mit der Erschöpfung durch Arbeit. Diese Erschöpfung kann verschiedenste Erscheinungsformen annehmen, immer sind es chronische, das Individuum schwer schädigende Formen von Destruktivität und Selbstentfremdung.

Der Autor beschäftigt sich mit der Auswirkung der Persönlichkeit des Menschen auf seinen Umgang mit der Arbeit. Dabei bezieht er das Verhältnis zu Arbeitskollegen, Vorgesetzten und Mitarbeitern mit ein, denn wir alle gehen sehr unterschiedlich mit den Realitäten der Arbeitswelt um. Burnout, Mobbing, Selbstentfremdung, aber auch die Lust an der Arbeit sind Stichworte, hinter denen sich eine tiefgehende Psychodynamik verbindet.

Prof. Dr. Karl Könik ist Psychoanalytiker und Internist, Lehranalytiker und Supervisor am Göttinger Lou Andreas Salomé-Institut und dessen Leiter. 